\title{
Study of optical and luminescence properties of silicon - semiconducting silicide - silicon multilayer nanostructures
}

\author{
N.G. Galkin, K.N. Galkin*, S.A. Dotsenko, D.L. Goroshko, A.V. Shevlyagin, \\ E.A. Chusovitin, and I.M. Chernev \\ Institute of Automation and Control Processes FEB RAS, 690041 Vladivostok, Russia
}

\begin{abstract}
By method of in situ differential spectroscopy it was established that at the formation of monolayer $\mathrm{Fe}, \mathrm{Cr}, \mathrm{Ca}, \mathrm{Mg}$ silicide and $\mathrm{Mg}$ stannide islands on the atomically clean silicon surface an appearance of loss peaks characteristic for these materials in the energy range of 1.1$2.6 \mathrm{eV}$ is observed. An optimization of growth processes permit to grow monolithic double nanoheterostructures (DNHS) with embedded $\mathrm{Fe}, \mathrm{Cr}$ and $\mathrm{Ca}$ nanocrystals, and also polycrystalline DNHS with $\mathrm{NC}$ of $\mathrm{Mg}$ silicide and $\mathrm{Mg}$ stannide and $\mathrm{Ca}$ disilicide. By methods of optical spectroscopy and Raman spectroscopy it was shown that embedded NC form intensive peaks in the reflectance spectra at energies up to $2.5 \mathrm{eV}$ and Raman peaks. In DNS with $\beta-\mathrm{FeSi} 2 \mathrm{NC}$ a photoluminescence and electroluminescence at room temperature were firstly observed.
\end{abstract}

Semiconductor monolithic and polycrystalline nanoheterostructures created on the base of embedded in silicon matrix the nanocrystals (NCs) of semiconducting silicides $\left(\beta-\mathrm{FeSi}_{2}\right.$, $\mathrm{CrSi}_{2}, \mathrm{~h}-\mathrm{CaSi}_{2}, \mathrm{Mg}_{2} \mathrm{Si}$ ), as well as $\mathrm{Mg}$ stannide-silicide NCs can have fundamentally new optical properties associated with confinement of electrons and excitons. It is important to develop and create new light emitting and detecting devices and thermoelectrical convertors within silicon complimentary metal - oxide - semiconductor (CMOS) technology. Twoand three-layer structures with embedded $\beta-\mathrm{FeSi}_{2}$ precipitates $(100-150 \mathrm{~nm})$ in silicon with p-n junction were previously grown [1-3], as well as discovered and studied their electroluminescent properties. Decrease of $\beta-\mathrm{FeSi}_{2} \mathrm{NCs}$ sizes down to $40-60 \mathrm{~nm}$ and an increase in the number of layers embedded into silicon, have shown promise of this approach in terms of increasing the efficiency of photo- and electroluminescence in grown structures $[4,5]$. To reduce the probability of mismatch dislocation formation one can use embedding in silicon the nanosize $(5-20 \mathrm{~nm})$ crystals of silicides $\left(\beta-\mathrm{FeSi}_{2}, \mathrm{CrSi}_{2}, \mathrm{Mg}_{2} \mathrm{Si}, \mathrm{h}-\right.$ $\mathrm{CaSi}_{2}$ ) and $\mathrm{Mg}$ stannide, when elastic adjustment of nanocrystal lattice to the silicon lattice or three-dimensional pseudomorphism is proposed. In this case, on the interface semiconductor silicide (stannide) nanocrystals - silicon can expect an absence or significant decrease of dangling bonds and, accordingly, carrier scattering centers and their nonradiative recombination as well as transport of carriers without additional scattering mechanisms.

\footnotetext{
${ }^{*}$ Corresponding author: galkin@iacp.dvo.ru
} 
In this paper systematically investigated the nucleation conditions of nanosize silicide's islands of few transition and alkaline earth metals, as well as stannide and magnesium stannide-silicide by in situ method of differential reflectance spectroscopy, electron Auger spectroscopy and electron energy loss spectroscopy. It has been established that the formation of nanosize semiconductor islands of semiconductor metal's silicides and stannide leads to peak's formation in the spectra of the change of the imaginary part of dielectric function (CIPDF) in the photon energy range 1.1-1.7 eV [6], which is associated with the formation of the interband transitions with great oscillator force. In case of island formation of metallic silicides a monotonic decrease of CIPDF with increasing photon photons is observed. An optimization of growth parameters has permitted to create monolithic and multilayer nanoheterostructures with embedded in silicon matrix the $\mathrm{Fe}, \mathrm{Cr}$ and $\mathrm{Ca}$ disilicide, $\mathrm{Mg}$ and $\mathrm{Ca}$ silicide as well as $\mathrm{Mg}$ stannide-silicide nanocrystals. By data of cross sectional transmittance electron microscopy it was shown that in monolithic nanoheterostructures (MNHS) an elastic embedding of compressed and extended $\beta-\mathrm{FeSi}_{2}$ and $\mathrm{CrSi}_{2} \mathrm{NCs}$ with $5-20 \mathrm{~nm}$ sizes is observed in the monocrystalline silicon without formation of mismatch dislocations [5,7]. In the case of embedding of $\mathrm{Mg}$ and $\mathrm{Ca}$ silicide and stannide nanocrystals $(5-20 \mathrm{~nm})$ in the polycrystalline and amorphous silicon matrix the crystalline structure with arbitrary orientation relatively a silicon matrix is observed. By methods of transmittance and reflectance optical spectroscopy it was established that in the reflectance spectrum the appreciable peaks at energies up to $2.5 \mathrm{eV}$ are formed at increasing of embedded NCs layer quantities [8,9], but in the Raman spectra the peaks correlated with ones of silicides and stannides are observed [10,11]. In MNHS with seven embedded $\beta$ $\mathrm{FeSi}_{2} \mathrm{NCs}$ and thin silicon interlayers $(10-12 \mathrm{~nm})$ the electroluminescence with 25 micro watt power emission in the wavelength range of $1500-1550 \mathrm{~nm}$ at $300 \mathrm{~K}$ was firstly observed that corresponds to suppression of non-radiative recombination centers, and also to appearance of the quantum Stark effect and formation of excitons with binding energy more than $3 / 2 \mathrm{kT}$. This intensive electroluminescence was observed at pumping current density of $0.7 \mathrm{~A} / \mathrm{cm}^{2}$, which is a record low value. Linear dependence of optical power emission versus pumping current density throughout the studied range showed the minimization of non-radiative channels for carrier's recombination in diode structures. The stressed structure of $\beta-\mathrm{FeSi}_{2}$ NCs with sizes lower $15 \mathrm{~nm}$ ensured the formation of type-I heterostructure with direct band gap light emission that allows to count on creating of highspeed silicon infrared light emitting diodes working for $300 \mathrm{~K}$.

This work was carried out with financial support from the Russian Science Foundation grant (No. 16-19-10241).

\section{References}

1. T. Suemasu, T. Fujii, K. Takakura, F. Hasegawa, Thin Sol. Film. 381, 209 (2001)

2. T. Suemasu et al., Appl. Phys. Lett. 79, 1804 (2001)

3. T. Sunokawa et al., Jap. J. Appl. Phys. 44, 3951 (2005)

4. N.G. Galkin et al., J. Nanosci. \& Nanotechn. 8, 527 (2007)

5. N.G. Galkin et al., Thin Solid Films 519, 8480 (2011)

6. S.A. Dotsenko et al., e-J. Surf. Sci. \& Nanotechnol. 4, 319 (2006)

7. N.G. Galkin et al., Appl Surf. Sci. 256, 7331 (2010)

8. N.G. Galkin et al., J. Phys.: Conds. Matt. 19, 506204 (2007)

9. N.G. Galkin et al., Technic. Phys. 55, 1036 (2010)

10. S.A. Dotsenko, Phys. Proced. 23, 41 (2012)

11. N.G. Galkin et al., Jap. J. Appl. Phys. 54, 07 JC06 (2015) 